\title{
Análise da violência doméstica contra a mulher em tempos de pandemia da COVID-19
}

\author{
Analysis of domestic violence against women in times of the COVID-19 pandemic
}

Análisis de la violencia doméstica contra las mujeres en tiempos de la pandemia COVID-19

Márcya Cândida Casimiro de Oliveira ${ }^{1 *}$, André Luís Belmiro Moreira Ramos ${ }^{1}$, Nathália de Oliveira Azevedo1, lanara Fabiana Ramalho Dias Alves ${ }^{1}$, Domennica Gomes Pecorelli1, Gilberto José Montaño Góes de Mendonça1 ${ }^{1}$, Amanda Apolori Tissiani ${ }^{1}$, Layza de Souza Chaves Deininger ${ }^{1}$.

\section{RESUMO}

Objetivo: Analisar a violência doméstica contra a mulher em tempos de pandemia COVID-19 descrita na literatura científica. Métodos: Trata-se de uma revisão integrativa realizada em seis etapas, utilizando a questão norteadora: "Qual o comportamento da violência contra a mulher frente à pandemia do COVID19?". O levantamento bibliográfico foi realizado nas plataformas BVS, SciELO, LILACS e MEDLINE nos meses de abril e maio de 2021, sendo obtido 3.183 artigos, que após os critérios estabelecidos, foram selecionados 14 estudos. Resultados: O distanciamento social cria as condições ideais para que os elementos da violência sejam ampliados e muitas vítimas tenham dificuldade em denunciar o agressor. $O$ aumento da violência tem graves repercussões físicas, mentais, reprodutivas, emocionais, de saúde e segurança para as mulheres e seus filhos. Como forma de combater esse tipo de violência, é necessário o apoio do Estado com maior agilidade, bem como seu papel no fortalecimento da segurança das mulheres em situação de vulnerabilidade. Considerações finais: Portanto, as denúncias devem ser incentivadas para combater a impunidade do agressor e buscar reduzir a subnotificação dos casos.

Palavras-chave: COVID-19, Gênero, Morte, Pandemia, Violência doméstica.

\begin{abstract}
Objective: To analyze domestic violence against women during the COVID-19 pandemic described in the scientific literature. Methods: This is an integrative review carried out in six stages, using the guiding question: "What is the behavior of violence against women in the face of the COVID-19 pandemic?". The bibliographic survey was carried out on the VHL, SciELO, LILACS and MEDLINE platforms in April and May 2021, obtaining 3,183 articles, which after the established criteria, 14 studies were selected. Results: Social distancing creates the ideal conditions for the elements of violence to be expanded and many victims find it difficult to report the aggressor. The rise in violence has serious physical, mental, reproductive, emotional, health and safety repercussions for women and their children. As a way to combat this type of violence, it is necessary the State's support with greater agility, as well as its role in strengthening the security of women in vulnerable situations. Final considerations: Therefore, complaints should be encouraged to combat the aggressor's impunity and seek to reduce underreporting of cases.
\end{abstract}

Keywords: COVID-19, Gender, Death, Pandemic, Domestic violence.

\footnotetext{
${ }^{1}$ Faculdade de Ciências Médicas da Paraíba / Afya, Cabedelo - PB.

*E-mail: marcyacasimiro@gmail.com
}

SUBMETIDO EM: 10/2021

ACEITO EM: 10/2021

PUBLICADO EM: 11/2021 


\section{RESUMEN}

Objetivo: Analizar la violencia intrafamiliar contra la mujer durante la pandemia COVID-19 descrita en la literatura científica. Métodos: Se trata de una revisión integradora realizada en seis etapas, utilizando la pregunta orientadora: “ ¿Cuál es el comportamiento de la violencia contra las mujeres frente a la pandemia COVID-19?". EI relevamiento bibliográfico se realizó en las plataformas BVS, SciELO, LILACS y MEDLINE en abril y mayo de 2021, obteniendo 3.183 artículos, que de acuerdo con los criterios establecidos, se seleccionaron 14 estudios. Resultados: El distanciamiento social crea las condiciones ideales para que se amplíen los elementos de la violencia y muchas víctimas tienen dificultades para denunciar al agresor. El aumento de la violencia tiene graves repercusiones físicas, mentales, reproductivas, emocionales, de salud y seguridad para las mujeres y sus hijos. Como forma de combatir este tipo de violencia, es necesario el apoyo del Estado con mayor agilidad, así como su rol en el fortalecimiento de la seguridad de las mujeres en situaciones de vulnerabilidad. Consideraciones finales: Por lo tanto, las denuncias deben ser incentivadas para combatir la impunidad del agresor y buscar reducir la subnotificación de casos.

Palabras clave: COVID-19, Género, Muerte, Pandemia, Violencia doméstica.

\section{INTRODUÇÃO}

A doença do novo coronavírus, a COVID-19, é responsável pela atual pandemia e alterou a rotina de parcela significativa da população em decorrência de sua elevada patogenicidade e objetivando reduzir índices da transmissão do patógeno, foram recomendadas as medidas de distanciamento social, as quais contribuíram para o aumento da violência doméstica, sobretudo contra as mulheres. No contexto de pandemia, a política do isolamento social é fundamental para conter a disseminação do vírus. Entretanto, esta medida é apontada como a principal agravante para o aumento da incidência de violência doméstica contra a mulher, haja vista que o núcleo desse tipo de violência é o ambiente familiar e, durante a pandemia, as mulheres permanecem mais tempo próximas dos seus agressores (MARQUES ES, et al., 2020; FÓRUM BRASILEIRO DE SEGURANÇA PÚBLICA (FBSP), 2020; REIS AP, et al., 2020).

A violência doméstica perpetrada contra a mulher é uma realidade social e abrange agressões de caráter patrimonial, moral, psicológico, sexual, físico e não são raros os casos que culminam em assassinato, que representa manifestação mais grave dessa violência. Dados também apontam um crescimento da violência letal em 22,2\% entre os meses março e abril de 2020 quando comparados ao mesmo período do ano de 2019, correspondentes aos meses da instalação do distanciamento social da COVID-19, culminando com 143 mulheres mortas. Apesar disso, as mulheres sentem-se desestimuladas a buscar ajuda frente à situação de violência sofrida, seja por medo ou pela dificuldade de fazer denúncias em tempos de pandemia, sobretudo nas famílias com renda insuficiente, o que contribui em definitivo, para as subnotificações dos casos e a perpetuação desse crime (MENEGHEL SN e PORTELLA AP, 2017; FBSP, 2020; MARQUES ES, et al., 2020).

Ainda entre os meses de março e abril de 2020, em relação ao mesmo período do ano anterior, observou-se crescimento de $27 \%$ das denúncias telefônicas da violência doméstica contra a mulher no Ligue-180, bem como crescimento dos chamados para a Polícia Militar no 190, sobretudo em São Paulo com aumento de $44,9 \%$ em relação ao mesmo período supracitado. Vários fatores contribuíram para esta realidade, todos associados ao aumento da vulnerabilidade da vítima, como a restrição aos serviços públicos que conferem apoio social, redução da interação com familiares e amigos, além de aumento do contato com o agressor que por muitas vezes possui a sensação de impunidade e, dessa forma, continua perpetuando esse crime (FBSP, 2020; MINISTÉRIO PÚBLICO DE SÃO PAULO, 2020; ALENCAR J, et al., 2020; MARQUES ES, et al., 2020).

Esses números estão longe de representar a realidade, já que a subnotificação é um fato que não pode ser ignorado e a sociedade ainda é omissa frente às situações de violência doméstica contra a mulher, sendo também desconhecidas todas as repercussões dessas formas de agressão, justificando a necessidade de novos estudos acerca da temática (ALENCAR J, et al., 2020; CORTES, LF, 2020). 
Desse modo, considerando o aumento da violência contra a mulher diante do contexto da pandemia pelo novo coronavírus, bem como buscando compreender os principais impactos desse crime na atual crise sanitária mundial, o presente estudo objetivou analisar a violência doméstica contra a mulher em tempos de pandemia da COVID-19 descrita na literatura científica.

\section{MÉTODOS}

Trata-se de um estudo do tipo Revisão Integrativa (RI) da literatura realizada nos meses de abril e maio de 2021 com o intuito de analisar e sumarizar as informações disponibilizadas por estudos científicos publicados, de modo sistemático e organizado, contribuindo para o aprofundamento do conteúdo investigado e construção de uma análise crítica acerca da temática abordada. O estudo foi desenvolvido em seis etapas: definição da questão norteadora, delimitação dos critérios de inclusão e exclusão, coleta de dados dos artigos selecionados anteriormente, avaliação crítica dos artigos selecionados, discussão e interpretação dos resultados e apresentação da síntese elaborada (MENDES KS, et al., 2008; SOUZA MT, et al., 2010).

A questão norteadora do presente estudo foi: "Qual o comportamento da violência doméstica contra a mulher frente à pandemia da COVID-19?". A coleta de dados foi realizada nas bases de dados e bibliotecas eletrônicas: Biblioteca Virtual em Saúde (BVS), Scientific Eletronic Library Online (SciELO), Literatura Latino-Americana em Ciências de Saúde (LILACS) e Medical Literature Analysis and Retrievel System Online (MEDLINE). Foram coletados dados de 2020 a 2021, tendo em vista o período do início da pandemia. A busca dos artigos científicos foi realizada em duas etapas nas bases e bibliotecas referidas anteriormente, utilizado os Descritores em Ciências da Saúde (DeCs) e os booleanos AND e OR, de modo que, na primeira etapa, os descritores foram dispostos na seguinte combinação: violência doméstica AND morte AND COVID-19, chamados de D1; já na segunda etapa, a combinação: violência doméstica AND gênero AND COVID-19 OR pandemia, chamados de D2.

Os critérios de inclusão estabelecidos para a pesquisa foram: artigos científicos que contemplassem o objetivo da presente revisão integrativa, assim como estudos disponíveis na íntegra nos idiomas português, inglês e espanhol publicados nos anos 2020 e 2021. Como critérios de exclusão, foram estabelecidos: teses, monografias, dissertações, artigos duplicados, materiais não disponíveis na íntegra e que fugissem ao tema, além de recursos não científicos. Posteriormente a busca dos artigos, procedeu-se à leitura dos títulos e resumos, sendo que alguns já foram excluídos nesse momento. Todos os estudos enquadrados nos critérios de inclusão foram lidos na íntegra para seleção daqueles de maior relevância para a temática em questão. A seguir, os estudos foram classificados de acordo com o nível de evidência (MELNYK BM, 2005) (Quadro 1).

Quadro 1 - Níveis de evidência.

\begin{tabular}{|c|c|}
\hline Nível de evidência & Tipo de estudo \\
\hline I & $\begin{array}{l}\text { Revisão sistemática ou meta-análise de ensaios clínicos } \\
\text { randomizados. }\end{array}$ \\
\hline II & $\begin{array}{l}\text { Experimentos de pelo menos um ensaio clínico randomizado ou } \\
\text { controlado bem delineado. }\end{array}$ \\
\hline III & Experimentos controlados bem delineados sem randomização. \\
\hline IV & Estudo de coorte ou caso-controle bem delineados. \\
\hline $\mathrm{V}$ & Revisão sistemática de estudos qualitativos ou descritivos. \\
\hline VI & Estudos qualitativos ou descritivos. \\
\hline VII & $\begin{array}{c}\text { Opinião de autoridades e/ou relatórios de comitê de } \\
\text { especialistas. }\end{array}$ \\
\hline
\end{tabular}

Fonte: Oliveira MCC, et al., 2021. Baseado em Melnyk BM, 2005.

Para sistematização dos estudos selecionados, elaborou-se um fluxograma com os critérios estabelecidos no presente artigo (Figura 1). 
Figura 1 - Fluxograma dos critérios de inclusão e exclusão dos artigos.

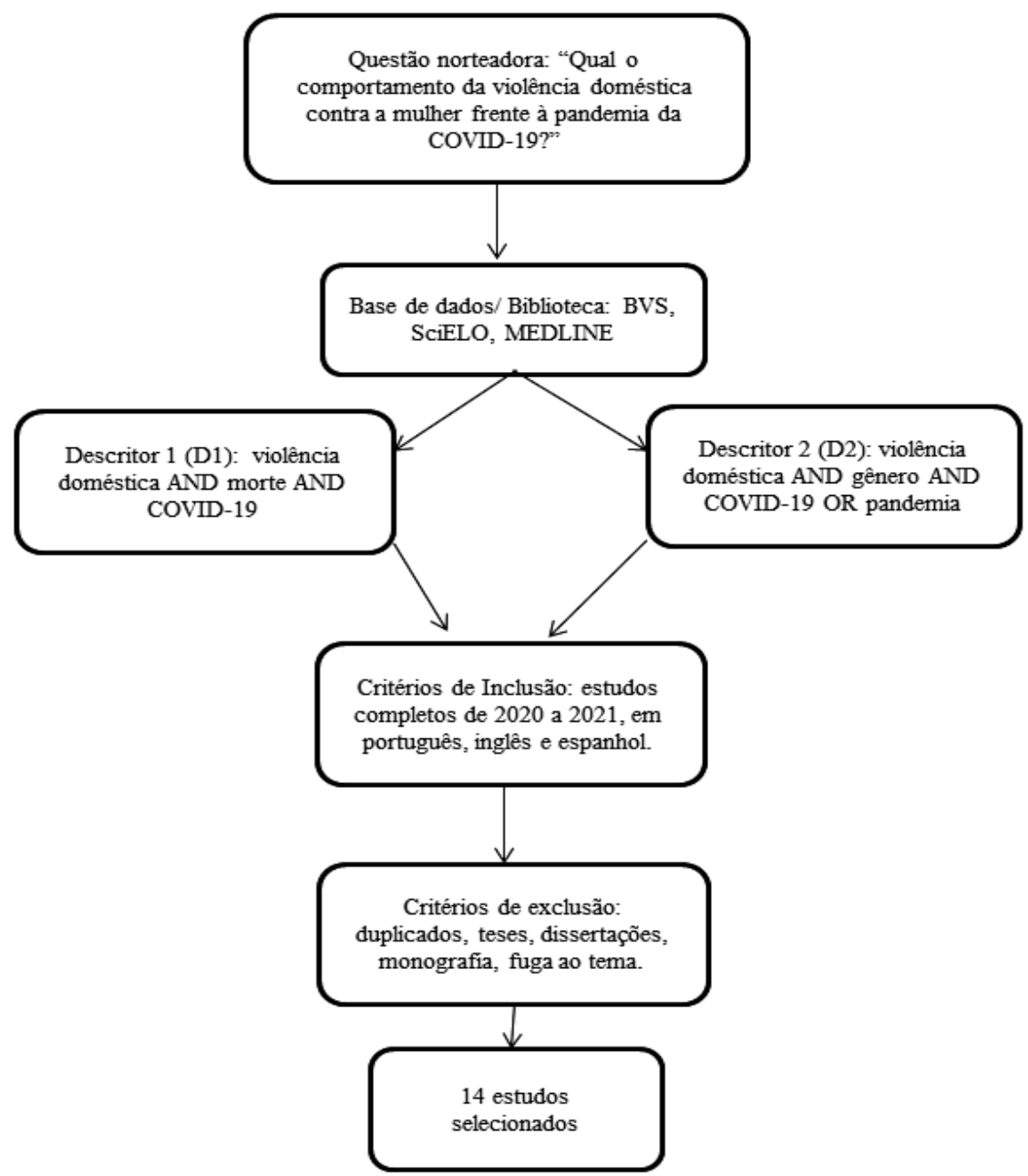

Fonte: Oliveira MCC, et al., 2021.

\section{RESULTADOS}

Na BVS, utilizando a combinação de descritores: violência doméstica AND morte AND COVID-19 foram encontrados 13 estudos, após o filtro restaram seis. Destes, apenas dois foram selecionados, sendo ambos em espanhol. Já com a combinação de descritores: violência doméstica AND gênero AND COVID-19 OR pandemia, foram encontrados 2.192 estudos, mas após a aplicação do filtro norteado pelos critérios de inclusão e exclusão, restaram 88. Após uma análise criteriosa, nove se enquadraram nos objetivos do presente estudo e foram selecionados, sendo sete no idioma inglês, já em português e espanhol, um estudo em cada idioma respectivamente.

$\mathrm{Na}$ SciELO, na primeira etapa da pesquisa, utilizando os descritores selecionados, nenhum estudo foi encontrado. Para a segunda etapa, foram encontrados 255 estudos, após a aplicação do filtro, restaram 40. Todavia, quatro eram duplicados e os demais não contemplavam o objetivo de estudo do presente artigo, portanto foram excluídos. Já na LILACS, com a primeira combinação de descritores foram encontrados três estudos. Contudo, após análise, apenas um foi selecionado no idioma português. Com a segunda combinação de descritores, foram encontrados 723 estudos, após o filtro restaram 49. Após uma análise minuciosa, apenas dois foram selecionados por correlacionarem a violência doméstica contra a mulher com a atual pandemia, um no idioma português e o outro, espanhol. Por fim, na MEDLINE, não foram encontrados estudos com nenhuma das combinações de descritores.

Sendo assim, a partir dos critérios estabelecidos para a busca dos artigos, foram encontrados 3.183 estudos nas bases de dados e bibliotecas virtuais referidas. Após uma análise minuciosa, 14 se 
enquadraram nos parâmetros almejados. Portanto, esta foi a amostra final de artigos da presente revisão integrativa. No que tange aos níveis de evidência, o de maior expressão foi o nível VI, seguido pelo VII e V, com $71,43 \%, 21,43 \%$ e $7,14 \%$ respectivamente e os demais níveis não detiveram representatividade nesta revisão. No que tange às bases de dados/ biblioteca virtual, a BVS foi a de maior expressão nos resultados, seguida pela LILACS, haja vista que as demais não detiveram representatividade na construção desta revisão. Visando facilitar a inspeção e apresentação dos resultados, elaboraram-se as Figuras 2 e 3, que apontam o processo de obtenção dos artigos encontrados com base na combinação de descritores selecionados.

Figura 2 - Fluxograma da seleção dos artigos com base na combinação de descritores D1.

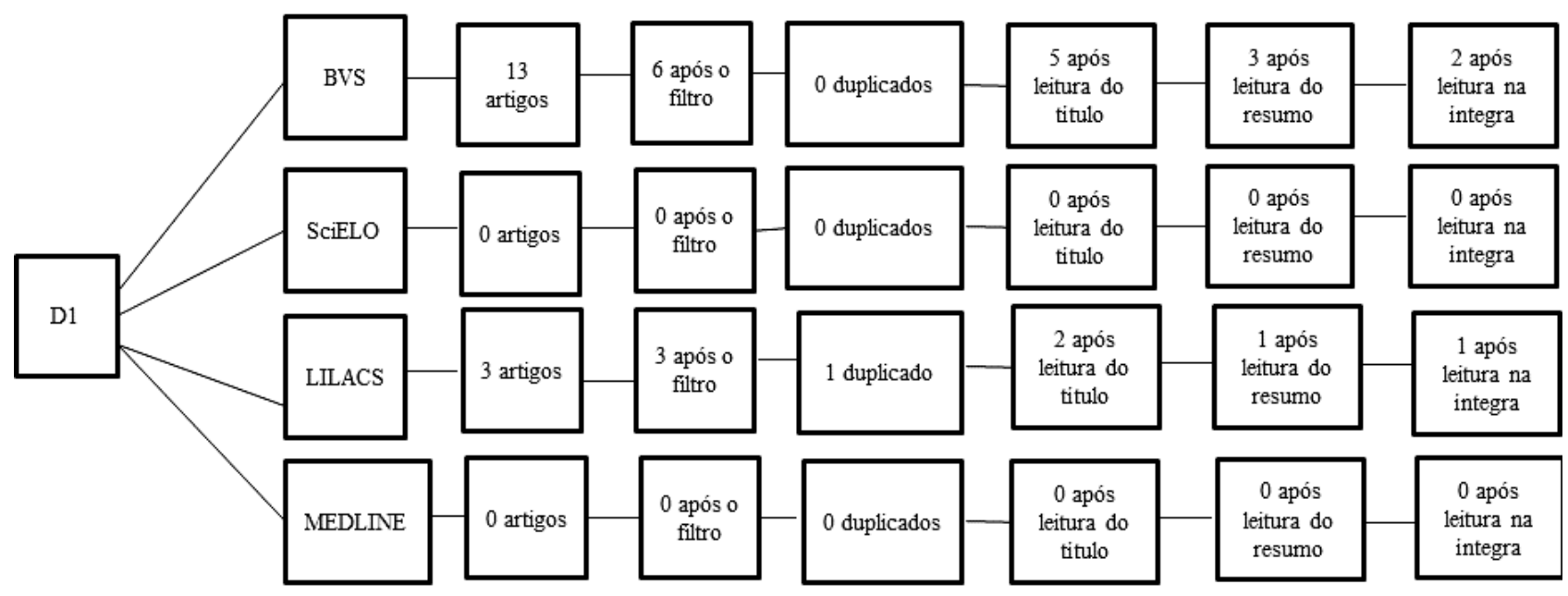

Total de artigos obtidos: $16 \quad$ Total de artigos excluidos: 16

Total de artigos selecionados: 3

Fonte: Oliveira MCC, et al., 2021.

Figura 3 - Fluxograma da seleção dos artigos com base na combinação de descritores D2.

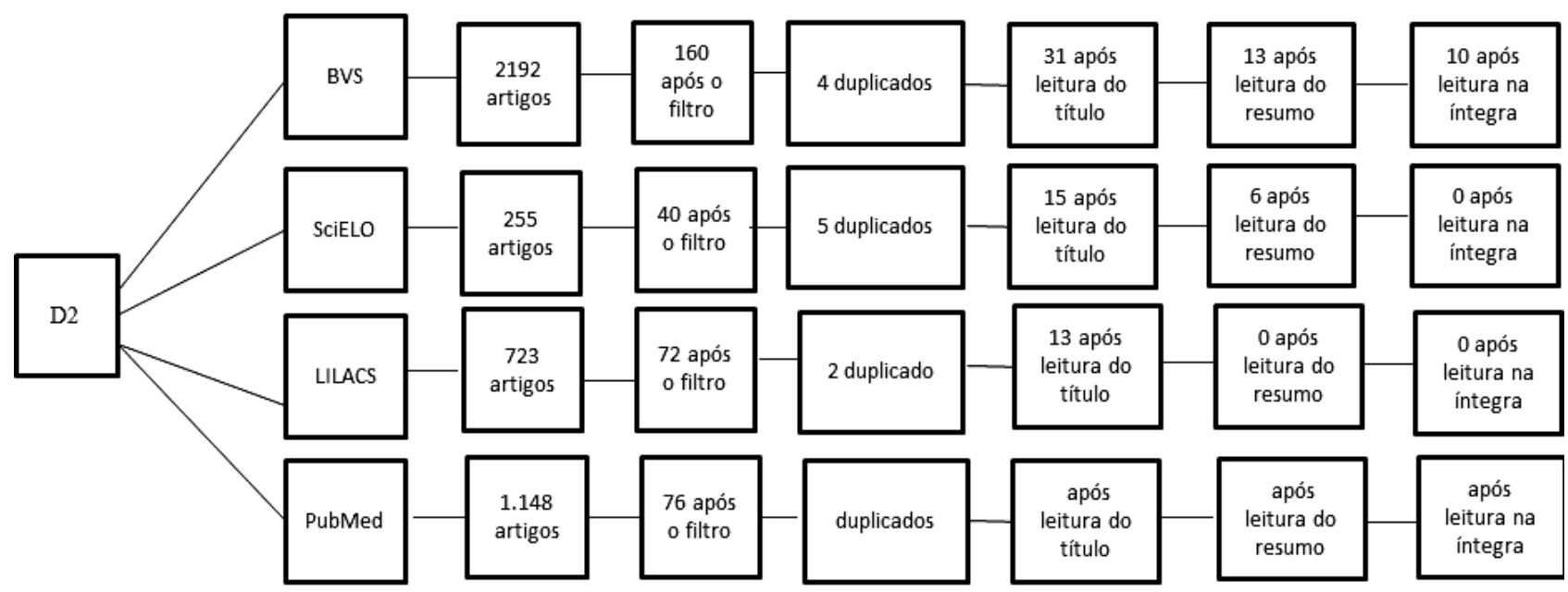

Total de artigos obtidos: $4.318 \quad$ Total de artigos excluídos: 4.307

Total de artigos selecionados: 11

Fonte: Oliveira MCC, et al., 2021.

Já o Quadro 2 aponta autores, base de dados, ano de publicação, nível de evidência, título e os principais resultados de cada estudo analisado, sendo D1 e D2 os estudos selecionados com a primeira e segunda combinação de descritores respectivamente (Quadro 2). 
Quadro 2 - Disposição dos resultados.

\begin{tabular}{|c|c|c|c|c|c|}
\hline Descritor & Autores/ Ano & $\begin{array}{l}\text { Base de dados/ } \\
\text { Biblioteca }\end{array}$ & $\begin{array}{l}\text { Nível de } \\
\text { evidência }\end{array}$ & Título do estudo & Principais resultados \\
\hline D1 & $\begin{array}{l}\text { LORENTE- } \\
\text { ACOSTA M, } 2020\end{array}$ & BVS & VI & $\begin{array}{l}\text { Violencia de género en tiempos de } \\
\text { pandemia y confinamento }\end{array}$ & $\begin{array}{l}\text { Durante o isolamento, houve aumento em todas as suas } \\
\text { formas de violência (física, psicológica e sexual). }\end{array}$ \\
\hline D1 & $\begin{array}{l}\text { RUIZ-PÉREZ I e } \\
\text { PASTOR- } \\
\text { MORENO G, } 2020\end{array}$ & BVS & $\mathrm{VI}$ & $\begin{array}{l}\text { Medidas de contención de la violencia } \\
\text { de género durante la pandemia de } \\
\text { COVID-19 }\end{array}$ & $\begin{array}{l}\text { Crises, emergências e momentos de agitação têm sido } \\
\text { associados a um aumento da violência interpessoal, } \\
\text { incluindo a violência contra as mulheres. }\end{array}$ \\
\hline D1 & $\begin{array}{l}\text { CORTES LF, et al., } \\
\qquad 2020\end{array}$ & LILACS & VI & $\begin{array}{l}\text { Desafios na proteção às mulheres em } \\
\text { situação de violência no contexto de } \\
\text { pandemia da COVID-19 }\end{array}$ & $\begin{array}{l}\text { O contexto da pandemia implicou o convívio prolongado } \\
\text { com parceiros, possibilitando que a violência contra as } \\
\text { mulheres intensificasse. }\end{array}$ \\
\hline D2 & $\begin{array}{l}\text { ALVAREZ- } \\
\text { HERNANDEZ; LR } \\
\text { et al., } 2021\end{array}$ & BVS & VI & $\begin{array}{l}\text { COVID-19 Pandemic and Intimate } \\
\text { Partner Violence: an Analysis of Help- } \\
\text { Seeking Messages in the Spanish- } \\
\text { Speaking Media. }\end{array}$ & $\begin{array}{l}\text { Os índices da violência contra a mulher por parceiros } \\
\text { íntimos aumentaram drasticamente com } 0 \text { isolamento } \\
\text { social. }\end{array}$ \\
\hline D2 & $\begin{array}{l}\text { REIS AP, et al., } \\
2020\end{array}$ & BVS & V & $\begin{array}{l}\text { Gender and race inequalities in the } \\
\text { COVID-19 pandemic: implications for } \\
\text { control in Brazil }\end{array}$ & $\begin{array}{l}\text { O distanciamento social tem levado várias mulheres a } \\
\text { ficarem confinadas com seus agressores, o que dificulta } \\
\text { o acesso aos serviços de denúncia, aumentando a } \\
\text { violência de gênero e graves repercussões para a saúde. }\end{array}$ \\
\hline D2 & XUE J, et al., 2020 & BVS & VI & $\begin{array}{l}\text { The Hidden Pandemic of Family } \\
\text { Violence During COVID-19: } \\
\text { Unsupervised Learning of Tweets. }\end{array}$ & $\begin{array}{l}\text { Faltam dados sobre as consequências do COVID-19 na } \\
\text { violência familiar }\end{array}$ \\
\hline D2 & $\begin{array}{l}\text { SANTOS LSE, et } \\
\quad \text { al., } 2020\end{array}$ & BVS & VI & $\begin{array}{l}\text { Impacts of the COVID-19 pandemic on } \\
\text { violence against women: reflections } \\
\text { from the theory of human motivation } \\
\text { from Abraham Maslow }\end{array}$ & $\begin{array}{l}\text { A pandemia da COVID-19 pode afetar a mulher que } \\
\text { sofre violência doméstica em todos os níveis } \\
\text { hierárquicos. }\end{array}$ \\
\hline
\end{tabular}




\begin{tabular}{|c|c|c|c|c|c|}
\hline Descritor & Autores/ Ano & $\begin{array}{l}\text { Base de dados/ } \\
\text { Biblioteca }\end{array}$ & $\begin{array}{l}\text { Nível de } \\
\text { evidência }\end{array}$ & Título do estudo & Principais resultados \\
\hline $\mathrm{D} 2$ & $\begin{array}{l}\text { SANTOS DF, et al., } \\
2020\end{array}$ & BVS & VI & $\begin{array}{l}\text { Masculinity in pandemic times: where } \\
\text { power decreases, violence increases }\end{array}$ & $\begin{array}{l}\text { Observa-se o aumento da violência domiciliar na } \\
\text { tentativa de estabilizar o modelo de masculinidade } \\
\text { definido pelo poder patriarcal. }\end{array}$ \\
\hline D2 & $\begin{array}{l}\text { BARBOSA JPM, et } \\
\text { al., } 2020\end{array}$ & BVS & VI & $\begin{array}{l}\text { Intersectionality and other views on } \\
\text { violence against women in times of } \\
\text { pandemic by COVID-19 }\end{array}$ & $\begin{array}{l}\text { Deve-se buscar entender como os diversos fluxos e } \\
\text { atravessam as mulheres em tempos de pandemia pela } \\
\text { COVID-19. }\end{array}$ \\
\hline $\mathrm{D} 2$ & PAHO, 2020 & BVS & VII & $\begin{array}{l}\text { COVID-19 y violencia contra la mujer. } \\
\text { Lo que el sector y el sistema de salud } \\
\text { pueden hacer }\end{array}$ & $\begin{array}{l}\text { A violência contra as mulheres, especialmente a } \\
\text { violência praticada pelo parceiro íntimo e a violência } \\
\text { doméstica, tem efeitos importantes na saúde das } \\
\text { mulheres e de seus filhos. }\end{array}$ \\
\hline $\mathrm{D} 2$ & WHO, 2020 & BVS & VII & $\begin{array}{l}\text { Addressing violence against children, } \\
\text { women and older people during the } \\
\text { COVID-19 pandemic: key actions }\end{array}$ & $\begin{array}{l}\text { A capacidade reduzida de obter ajuda durante a } \\
\text { pandemia COVID- } 19 \text { é provável que seja semelhante } \\
\text { para pessoas mais velhas sofrendo de abuso e } \\
\text { negligência. }\end{array}$ \\
\hline D2 & $\begin{array}{l}\text { ALENCAR J, et al., } \\
2020 .\end{array}$ & BVS & VII & $\begin{array}{l}\text { Políticas Públicas e Violência Baseada } \\
\text { no Gênero Durante a Pandemia Da } \\
\text { Covid-19: Ações Presentes, Ausentes } \\
\text { e Recomendadas }\end{array}$ & $\begin{array}{l}\text { Violência contra mulheres é uma violação de caráter } \\
\text { cultural e estrutural. Portanto, seu enfrentamento } \\
\text { também é complexo e deve ser multisetorial. }\end{array}$ \\
\hline $\mathrm{D} 2$ & IETSI, 2020 & LILACS & $\mathrm{VI}$ & $\begin{array}{l}\text { Violencia de género/familiar en } \\
\text { tiempos de cuarentena: revisión crítica } \\
\text { de la literature }\end{array}$ & $\begin{array}{l}\text { O isolamento social vem reforçando vulnerabilidades e } \\
\text { gerando vítimas com diferentes tipos de violência. }\end{array}$ \\
\hline D2 & $\begin{array}{l}\text { LIRA MOSC, et al., } \\
2020\end{array}$ & LILACS & VI & $\begin{array}{l}\text { Repercussões da COVID-19 no } \\
\text { cotidiano da mulher: reflexões sob o } \\
\text { olhar sociológico de Michel Maffesoli }\end{array}$ & $\begin{array}{l}\text { Foram previstas repercussões econômicas, emocionais, } \\
\text { na segurança e autonomia da mulher, em decorrência da } \\
\text { COVID-19. }\end{array}$ \\
\hline
\end{tabular}

Fonte: Oliveira MCC, et al., 2021. 


\section{DISCUSSÃO}

Observou-se que com o isolamento social da pandemia houve aumento de todas as formas de violência contra a mulher, como a emocional, psicológica, sexual e física, já que o distanciamento social imposto pelas autoridades sanitárias levou várias mulheres a ficarem mais tempo com seus agressores, revelando condições ideais para que os elementos da violência sejam ampliados, posto que isola ainda mais as mulheres, aumenta o controle do agressor, contribui para impunidade ao aumentar as barreiras para as denúncias, bem como cria um contexto que facilita o uso de qualquer uma de suas formas ao menor estímulo, com consequências nefastas para a saúde física, mental e emocional para a mulher e, por vezes, para seus filhos, que sofrem com a falta de segurança, baixo autoestima e desenvolvimentos de sintomas psiquiátricos (ALENCAR J, et al., 2020; INSTITUTO DE EVALUACIÓN DE TECNOLOGÍAS EN SALUD E INVESTIGACIÓN (IETSI), 2020; LORENTE-ACOSTA M, 2020; PAN AMERICAN HEALTH ORGANIZATION (PAHO), 2020; REIS AP, et al. 2020; LIRA MOSC, et al., 2020).

Verificaram-se fatores de riscos associados ao aumento da violência doméstica, com destaque para o uso excessivo de álcool e drogas, dificuldades financeiras e desemprego, relacionamentos abusivos e a tentativa de perpetuar o poder patriarcal. No tocante a sujeição da mulher a esse tipo de relacionamento, a dependência financeira do público feminino e, em muito caos, a vigilância constante do parceiro sobre sua individualidade, monitorando e controlando o acesso à internet e as ligações efetuadas e/ou recebidas são pontos dificultam a denúncia e ajudam a perpetuar a subnotificação e a impunidade. Além disso, existe um contexto histórico em torno da cultura machista e patriarcal, as quais são pautadas na submissão feminina, relações de inferioridade, dependência econômica por parcela das vítimas, além da associação da mulher como obrigação de cumprir as funções domésticas (SANTOS DF, et al., 2020; XUE J, et al., 2020; WORLD HEALTH ORGANIZATION (WHO), 2020; IETSI, 2020).

Observou-se que, além da sensação de impunidade, a conjuntura histórica em torno da figura do homem contribui para o aumento da violência doméstica contra a mulher, já que corriqueiramente o sexo masculino é visto como referência nas decisões e manutenção da ordem. É relevante anotar que a pandemia trouxe uma crise econômica e nem todos estavam preparados para enfrentá-la. Desse modo, a instabilidade econômica pode ser interpretada como perda do poder masculino, sobretudo quando este é o principal provedor do lar e essa situação tende a gerar ainda mais comportamentos controladores, violentos e agressivos (LORENTE-ACOSTA M, 2020; VIEIRA PR, et al., 2020).

Além disso, o isolamento social dificulta o acesso aos serviços de denúncia e esse empecilho contribui para o aumento dos índices dessa violência, a qual é causadora de graves repercussões para a saúde. Logo, a maioria dos casos não é reportada e nem sempre é possível buscar ajuda. Revelou-se também que a atual pandemia diminuiu o contato com outras pessoas do convívio diário, impossibilitando colegas de trabalho e amigos de sentirem mudanças de comportamento e perceberem as lesões físicas provocadas pelo agressor, afastando a mulher de sua rede de apoio social, destacando que a violência é heterogênea, apresentando-se de formas diferentes em grupos sociais distintos, sendo mais proeminente nos grupos de mulheres de maior vulnerabilidade e desigualdade social e econômica (ALENCAR J, et al., 2020; ALVAREZHERNANDEZ LR, et al., 2021; REIS AP, et al., 2020; RUIZ-PÉREZ I e PASTOR-MORENO G, 2020; XUE J, et al., 2020; UN WOMEN, 2020).

Assim, o atual contexto aponta para a necessidade de proteção das mulheres, especialmente as em condições de maior vulnerabilidade, visto que não é raro que a violência doméstica perpetrada contra essas pessoas permaneça na invisibilidade social. É fato que barreiras existem e dificultam a busca por uma rede de apoio, mas precisam ser superadas (CORTES LF, et al., 2020; MASCARENHAS MMDM, et al., 2020).

Outro problema observado foi à percepção por parte dos agressores em relação à sua vítima. Quando eles sentiam perda de controle sobre a mulher, aumentavam os riscos da violência doméstica, podendo levar, inclusive, ao óbito da vítima. Enfatiza-se que essa violência pode ser praticada por familiares e pelos parceiros do sexo masculino, sendo este último caso o de maior prevalência. Como consequência, o isolamento social gerou reclusão da mulher com o seu agressor, impossibilitando um ambiente seguro para a vítima que necessitava se resguardar em casa como medida preventiva imprescindível da COVID-19 (LORENTE-ACOSTA M, 2020; IETSI, 2020; REIS AP, et al., 2020; SANTOS LSE, et al., 2020). 
São inúmeros os efeitos da violência doméstica contra a mulher. Dentre as principais consequências, pode-se evidenciar a ansiedade, medo, sentimento de inferioridade, baixa autoestima, insegurança, falta de apoio econômico, além dos impactos físicos e mentais, entretanto, ainda faltam dados sobre todas as repercussões da COVID-19 sobre a violência perpetrada no contexto familiar. Para compreendê-las, é necessário considerar a presença de casos da violência doméstica contra a mulher antes do isolamento social como um problema de prevalência significativa que, lamentavelmente, continua omissa em muitas situações (IETSI, 2020; LORENTE-ACOSTA M, 2020; XUE J, et al., 2020).

Mulheres sujeitas à violência doméstica vivem sobre forte estresse, já que, além de lidar com o medo constante de agressões e os afazeres domésticos, ainda possuem forte propensão em contraírem o novo coronavírus quer seja no ambiente de trabalho ou no próprio lar, estando mais suscetível ao desenvolvimento de doenças psicológicas. Constatou-se ainda que, em momentos de epidemias, mulheres com idade mais avançada ou com algum tipo de deficiência ficam mais vulneráveis para sofrerem violência, causando impactos relevantes à saúde como um todo (REIS AP, et al., 2020; PAHO, 2020; WHO, 2020).

Enfatiza-se que em cenários de crises e emergências, sejam eles de cunho político, social, econômico ou da saúde, costumam ter associação com o crescimento da violência entre as pessoas, abrangendo, inclusive, a violência contra as mulheres. Tal realidade representa mais um indicativo preocupante da falta de segurança e que carece de resolução. Corroborando esses dados, países como Brasil, China, Colômbia, México, Austrália, Chipre, Estados Unidos e França relataram aumento importante da violência doméstica contra a mulher em tempos de pandemia da COVID-19, demonstrando não ser uma condição pontual (CORTES LF, et al., 2020; RUIZ-PÉREZ I e PASTOR-MORENO G, 2020).

Por outro lado, percebe-se diminuição dos boletins de ocorrência da violência de gênero (SANTOS LSE, et al., 2020). Todavia, mesmo com menos notificações, os casos de feminicídio lamentavelmente elevaramse após a instalação do distanciamento em 12 estados brasileiros, sendo o Acre, Maranhão e Mato Grosso os locais com maiores índices desse aumento no país, que soma ao todo 3.800 mulheres vítimas deste crime em 2019, embora seja pacífico que os números reais são maiores que os oficiais em decorrência da subnotificação dos casos (FBSP, 2020).

No atual cenário, o declínio nas notificações encontra explicação no contato mais próximo com o agressor e pela dificuldade de acessar os locais de denúncias, favorecendo a subnotificação dos casos, embora este seja um problema com raízes anteriores à atual pandemia. Apesar desse cenário grave, iniciativas e medidas foram criadas em vistas a reduzir os casos de violência durante a pandemia da COVID-19, a exemplo da Espanha, que estabeleceu o atendimento integral às vítimas de violência de gênero, com ênfase na mulher em situação de risco, contando com apoio psicológico, jurídico e social tanto pelo telefone, como pelos demais canais de denúncias. Nesses casos, ainda existe possibilidade de profissionais de saúde e vítimas compartilharem a sua localização para que possam receber apoio imediato das Forças de Segurança do Estado (BARBOSA JPM, et al., 2020; REIS AP, et al., 2020RUIZ-PÉREZ I e PASTOR-MORENO G, 2020).

Como forma de combate a esse tipo de violência, necessita-se do apoio do Estado com mais celeridade, bem como se destaca o papel diante do reforço para a segurança da mulher em condição de vulnerabilidade. Seria promissor que essas mulheres pudessem contar com a contribuição de uma rede de apoio em plataformas virtuais como garantia de comunicação e suporte frente ao cenário do distanciamento social. Contudo, não é medida simples de se implantada, já que muitas mulheres ainda não têm acesso à internet ou possuem suas atividades monitoradas pelo seu agressor (LORENTE-ACOSTA M, 2020; IETSI, 2020; WHO, 2020).

No momento atual da pandemia, é imprescindível fortalecer ações multissetoriais, com ênfase nos três poderes do Estado, bem como nas iniciativas civis e privadas de modo a assegurar assistência integral à vítima e, desse modo, tentar reduzir o crescente número de casos desse tipo de violência. Logo, frente ao crescente aumento da violência contra a mulher, é necessário amparar especialmente aquelas mais vulneráveis a esse crime, fornecendo suporte para que essas vítimas tenham sua segurança restaurada, sobretudo nos seus domicílios (ALENCAR J, et al., 2020). 
Diante da indiscutível importância do distanciamento social frente à COVID-19, o ambiente doméstico se configura como principal local de interação entre os familiares e, por isso, pautas devem ser levantadas diante do enfrentamento da atual pandemia. Dessa forma, trata-se de um desafio para a saúde e segurança da mulher no que tange ao combate da violência doméstica contra esse público alvo. Nessa perspectiva, enfatiza-se que a violência doméstica contra a mulher é parte da violência de gênero e representa um tipo de violência interpessoal. Em vista disso, o objetivo maior desse tipo de violência não é causar danos, mas provocar controle e submissão da vítima por parte do agente agressor (IETSI, 2020; LORENTE-ACOSTA M, 2020).

Destaca-se que, historicamente, a mulher foi posicionada de forma desigual e em condição de inferioridade, além de sofrer diferentes tipos de violência em vários âmbitos da vida, e nem sempre foram amparadas nesses momentos de fragilidade. Sendo assim, é preciso lutar pelo direito da mulher de ter assegurado sua integridade psicológica e física, especialmente durante a atual pandemia, uma vez que elas necessitam passar maior tempo no seu domicílio para o enfrentamento do novo coronavírus (ALENCAR J, et al., 2020).

\section{CONSIDERAÇÕES FINAIS}

Portanto, devido à dificuldade formalizar as denúncias é fundamental reforçar a segurança dessas mulheres com o fim de proporcionar proteção e amparo ao buscar o poder público, o que contribuiria para a redução da subnotificação dos casos, haja vista que o medo de delatar um caso de violência doméstica é uma realidade que contribui para a continuidade desse crime. Logo, canais de denúncia necessitam ser ampliados, com ênfase no fácil acesso, sobretudo para as mulheres em condições econômicas desfavoráveis e com renda insuficiente, de modo que a impunidade da violência doméstica contra a mulher seja combatida.

\section{REFERÊNCIAS}

1. ALENCAR J, et al. Políticas públicas e violência baseada no gênero durante a pandemia da Covid-19: ações presentes, ausentes e recomendadas. IPEA, Brasília. 2020. Disponível em: https://www.ipea.gov.br/portal/index.php?option=com_content\&view=article\&id=35884\#: :text=Nota\%20T\%C3\%A9c nica\%20\%2D\%202020\%20\%2D\%20Junho\%20\%2D,A\%C3\%A7\%C3\%B5es\%20Presentes\%2C\%20Ausentes\%20e \%20Recomendadas\&text=A\%20pandemia\%20do\%20novo\%20coronav\%C3\%ADrus,apresentado\%20m\%C3\%BAltip las\%20influ\%C3\%AAncias\%20na\%20sociedade. Acessado em: 19 abr. 2021.

2. ALVAREZ-HERNANDEZ LR, et al. COVID-19 Pandemic and Intimate Partner Violence: an Analysis of Help-Seeking Messages in the Spanish-Speaking Media. Journal of Family Violence, 2021; 1-12.

3. BARBOSA J, et al. Intersectionality and other views on violence against women in times of pandemic by COVID-19. Saúde e Sociedade, 2021; 30: 1-21.

4. CORTES LF. Desafios na proteção às mulheres em situação de violência no contexto de pandemia da COVID-19. Ciência, Cuidado e Saúde, 2020; 19: e27984.

5. FÓRUM BRASILEIRO DE SEGURANÇA PÚBLICA (FBSP). Violência doméstica durante a pandemia de Covid-19. 2020. Disponível em: https://forumseguranca.org.br/wp-content/uploads/2020/06/violencia-domestica-covid-19-ed02v5.pdf. Acessado em: 21 abr. 2021.

6. INSTITUTO DE EVALUACIÓN DE TECNOLOGÍAS EN SALUD E INVESTIGACIÓN (IETSI). Violencia de género/familiar en tiempos de cuarentena: Revicón crítica de la literatura. Reporte de resultados de investigación. 2020. Disponível em: https://docs.bvsalud.org/biblioref/2020/05/1096342/rri-08-2020-violencia-de-generofamiliar-entiempos-de-cuarentena.pdf. Acessado em: 10 de maio de 2021.

7. LIRA M, et al. Repercussões da COVID-19 no cotidiano da mulher: reflexões sob o olhar sociológico de Michel Maffesoli. Enfermagem em foco, 2020; 11(2): 231-235.

8. LORENTE-ACOSTA M. Violencia de género en tiempos de pandemia y confinamiento. Revista Española de Medicina Legal, 2020; 46(3): 139-145.

9. MARQUES E, et al. Violence against women, children, and adolescentes during the COVID-19 pandemic: overview, contributing factors, and mitigating measures. Cadernos de Saúde Pública, 2020; 36(4): 1-4.

10. MASCARENHAS M, et al. Análise das notificações de violência por parceiro íntimo contra mulheres, Brasil, 2011 2017. Revista brasileira de epidemiologia, 2020; 23(1): 2-11. 
11. MELNYK BM. Making the case for evidence-based practice. In: MELNYK B. M.; FINEOUT-OVERHOLT, E. Evidence based practice in nursing \& healthcare: a guide to best practice. Philadelphia: Lippincot Williams \& Wilkins, 2005; 24p.

12. MENDES K, et al. Revisão integrativa: método de pesquisa para a incorporação de evidências na saúde e na enfermagem. Texto contexto - enfermagem, 2008; 17(4): 758-764.

13. MENEGHEL SN, PORTELLA AP. Feminicídios: conceitos, tipos e cenários. Revista Ciência \& Saúde Coletiva, 2017; 22(9): 3077-3086.

14. MINISTÉRIO PÚBLICO DE SÃO PAULO (MPSP). RAIO X da violência doméstica durante isolamento: um retrato de $\begin{array}{llll}\text { São } & \text { Paulo. } 2020 . & \text { Disponível em: }\end{array}$ http://www.mpsp.mp.br/portal/page/portal/noticias/noticia?id_noticia=22511423\&id_grupo=11811. Acessado em: 11 mai. 2021.

15. PAN AMERICAN HEALTH ORGANIZATION (PAHO). COVID-19 y violencia contra la mujer. Lo que el sector y el sistema de salud hueden 2020.2 Disponível em: https://iris.paho.org/bitstream/handle/10665.2/52034/OPSNMHMHCovid19200008_spa.pdf?sequence=1\&isAllowed= y. Acessado em: 21 de abril de 2021.

16. REIS AP, et al. Gender and race inequalities in the Covid-19 pandemic: implications for control in Brazil. Saúde em Debate, 2021; 44: 324-340.

17. RUIZ-PEREZ, PASTOR- MORENO G. Medidas de contención de la violencia de género durante la pandemia de COVID-19. Gaceta Sanitaria, 2021; 35(4): 389-394.

18. SANTOS DF, et al. Masculinity in pandemic times: where power decreases, violence increases. Saúde e Sociedade, 2021; 30: e200535.

19. SANTOS LSE, et al. Intimate Partner Violence during the COVID-19 Pandemic: A Review of the Phenomenon from Victims and Help Professionals Perspectives. International Journal of Environmental Research and Public Health, $2021 ; 18(12): 6204$.

20. SOUZA M, et al. Revisão integrativa: o que é e como fazer. Einstein, 2010; 8(1): 102-106.

21. UN WOMEN. Strategies for the prevention of violence against women in the context of COVID-19 in Latin America and the Caribbean. 2020. Disponível em: https://lac.unwomen.org/en/digiteca/publicaciones/2020/04/prevencion-dela-violencia-contra-las-mujeres-frente-a-covid-19. Acessado em: 11 de maio de 2021.

22. VIEIRA P, et al. Isolamento social e o aumento da violência doméstica: o que isso nos revela? Revista Brasileira de Epidemiologia, 2020; 23: e200033.

23. WORLD HEALTH ORGANIZATION (WHO). Addressing violence against children, women and older people during the COVID-19 pandemic: key actions. 2020. Disponível em: https://apps.who.int/iris/handle/10665/332458. Acessado em: 11 de maio de 2021.

24. XUE J, et al. The Hidden Pandemic of Family Violence During COVID-19: Unsupervised Learning of Tweets. Journal of Medical Internet Research, 2020; 22(11): e24361. 